\title{
Association between severe sarcopenic obesity and respiratory incidence and mortality: an obesity paradox.
}

Introduction - Obesity remains one of the biggest health challenges worldwide. Sarcopenia, a progressive loss of muscle strength, is associated with a higher risk of disability and lower quality of life. Both conditions can occur independently of each other; however, share a common inflammatory pathway, leading to serious health problems. Previous studies have shown a positive association between severe sarcopenia and respiratory disease incidence/mortality, however, it is unclear if this association is modified by obesity. The aim of this work, therefore, was to investigate the association of severe sarcopenia and severe sarcopenic-obesity with respiratory incidence and mortality in the UK Biobank cohort.

Material and methods - 242,572 white participants from the UK biobank study were included. Severe sarcopenia was defined as the combination of low muscle mass, low grip strength and slow gait speed. Severe sarcopenic-obesity was defined, using 3 different criteria. The combination of severe sarcopenia plus at least one of the following criteria: BMI $\geq 30 \mathrm{~kg} / \mathrm{m}^{2}$, waist circumference $(\mathrm{WC})>$ $88 \mathrm{~cm}$ in women and $>102 \mathrm{~cm}$ in men, or the two highest quintiles of body fat $(60 \%)$. Associations between severe sarcopenic and severe sarcopenic-obesity and respiratory incidence and mortality were investigated using Cox-proportional hazard models.

Results - In people without sarcopenia, high BMI, WC and body fat were associated with a reduced risk of respiratory disease mortality (HR: 0.70 [0.52; 0.85], HR: 0.74 [95\%CI: 062: 088] and HR: 0.74 [95\%CI: 0.63 ; 0.88], respectively). In comparison to people without sarcopenia or obesity, those with severe sarcopenia had three times higher risk of respiratory disease incidence (HR: 3.13 [95\% CI: 2.25; 4.35]) and five times higher risk of mortality (HR: 5.37 [95\% CI: 2.96: 9.74]). However, sarcopenic-obesity, based on WC and body fat, was only associated with a moderately increased respiratory disease incidence (HR 1.60 [95\%CI: 1.04; 2.46$]$ and HR: 1.52 [1.04: 2.22], respectively). There were no associations between respiratory mortality and sarcopenic-obesity.

Discussion - Higher levels of adiposity may be a protective factor against respiratory mortality and could reduce the effect of severe sarcopenia over this disease. However, the mechanism behind this association needs to elucidate.

\section{Conflict of Interest}

There is no conflict of interest 